\title{
The Development of Microcalorimeter EDS Arrays
}

\author{
K.D. Irwin,* J. A. Beall,* S. Deiker,* G. C. Hilton,* L. King, ** S. W. Nam, ${ }^{*}$ D. E. Newbury, ** C. \\ D. Reintsema,* J. A. Small,** and L. R. Vale* \\ * Electronics and Electrical Engineering Laboratory, NIST, Boulder, CO 80305 \\ ** Chemical Science and Technology Laboratory, NIST, Gaithersburg, MD 20899
}

High-energy-resolution cryogenic microcalorimeters are a powerful new tool for x-ray microanalysis. ${ }^{1}$ With demonstrated energy resolution 20 times better than withconventional semiconductor EDS, microcalorimeters are useful in applications such as nanoscale particle analysis. Unfortunately, single x-ray microcalorimeters are limited by low count rate $\left(\sim 500 \mathrm{~s}^{-1}\right)$ and small area $\left(\sim 0.16 \mathrm{~mm}^{2}\right)$. Both the count rate and the area can be improved by the implementation of arrays of microcalorimeters. In principle, this improvement in count rate and area comes without degraded energy resolution. The implementation of small microcalorimeter arrays will lead to improvements in the minimum detectable size of nanoscale particles and in the trace element concentration detection limit for a fixed analysis time. The development of kilopixel arrays capable of acquiring hundreds of thousands of counts per second, and with collecting areas of order $50 \mathrm{~mm}^{2}$, will make it possible to collect high-statistics spectra in small fractions of a second. This will make new applications possible, including real-time process-stream monitoring and the study of the evolution of film properties during deposition with x-ray fluorescence.

Before fabricating an array, it is useful to demonstrate the operation of a singlepixel microcalorimeter system in different environments. We have now transferred a singlepixel microcalorimeter system from Boulder, Colorado to the Chemical Science and Technology Laboratory in Gaithersburg, Maryland. In the process of transferring the system, we discovered that our shadowmask-fabricated microcalorimeters based on aluminum-silver bilayers were not stable in the humid environment of Maryland. We now fabricate microcalorimeters photolithographically, using the immiscible molybdenum-copper material system, which we have found to be stable and robust. An example spectrum taken with the system in Gaithersburg, Maryland, is shownin Figure 1. The microcalorimeter is a $0.16 \mathrm{~mm}^{2}$ molybdenum-copper bilayer with a 3 - $\mu \mathrm{m}$-thick bismuth absorber. The energy resolution with real-time analog signal processing is $\sim 7 \mathrm{eV}$. This result compares well with the $\sim 4 \mathrm{eV}$ achieved with aluminum-silver bilayers, since the saturation energy of the new detectors is significantly higher. We will use molybdenum-copper bilayers in arrays.

In order to fabricate large arrays, it is necessary to develop an array structure that thermally isolates the adjacent pixels, while still allowing the leads to be extracted from the array to instrument the microcalorimeters. We fabricate single pixels on a silicon-nitride-coated silicon wafer. To create the needed thermal isolation, we remove the silicon beneath the microcalorimeter using a wet $\mathrm{KOH}$ etch, leaving the microcalorimeter on a suspended silicon-nitride membrane. Unfortunately, fabricating large arrays with thermally isolated pixels using this approach is difficult. To make it possible to fabricate large arrays, we are developing surface micromachining techniques to make arrays of silicon nitride platforms suspended over a silicon wafer using a sacrificial layer approach. A small array of such structures is shown in Figure 2. We are now developing the process to fabricate microcalorimeters on these platforms. 
Another difficulty in the development of kilopixel arrays is the implementation of the cryogenic wiring and electronics to instrument a large number of channels. The heat load and complexity involved in running wires from room temperature to every pixel is prohibitive. To overcome this difficulty, we are developing superconducting electronics to multiplex the readout of microcalorimeters at cryogenic temperatures. ${ }^{2}$ A 32-channel Superconducting Quantum Interference Device (SQUID) multiplexer chip is shown in Figure 3. While considerable work remans in the development of the cryogenic circuits and room-temperature electronics, in the future kilopixel arrays of microcalorimeters will be instrumented using only 32 SQUID multiplexer output channels.

\section{References:}

1. D. A. Wollman et al., J. Microscopy 188 (1997) 196.

2. J.A. Chervenak et al., Appl. Phys. Letters 74 (1999) 4043.

3. This work was supported in part by the NIST Office of Microelectronic Products (OMP). Contribution of the U.S. Government; not subject to copyright.

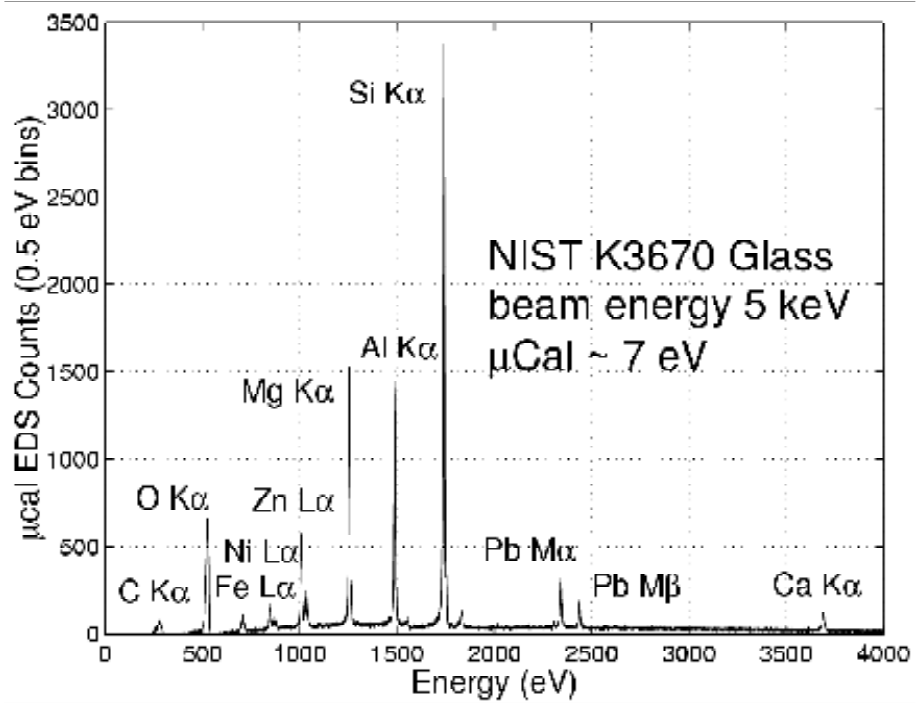

Figure 1. Microcalorimeter EDS spectrum of NIST calibration glass K3670 taken by the microcalorimeter system transferred to Gaithersburg, Maryland.

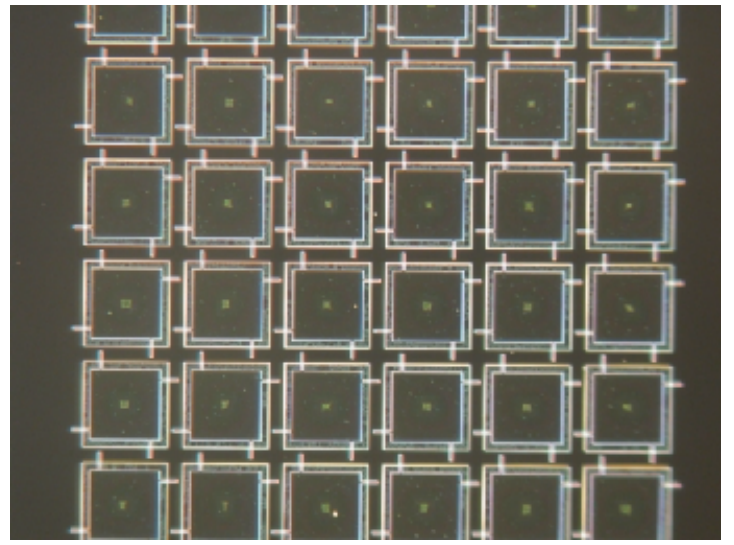

Figure 2. A 6x6 array of suspended silicon nitride platforms to thermally isolate separate microcalorimeter pixels

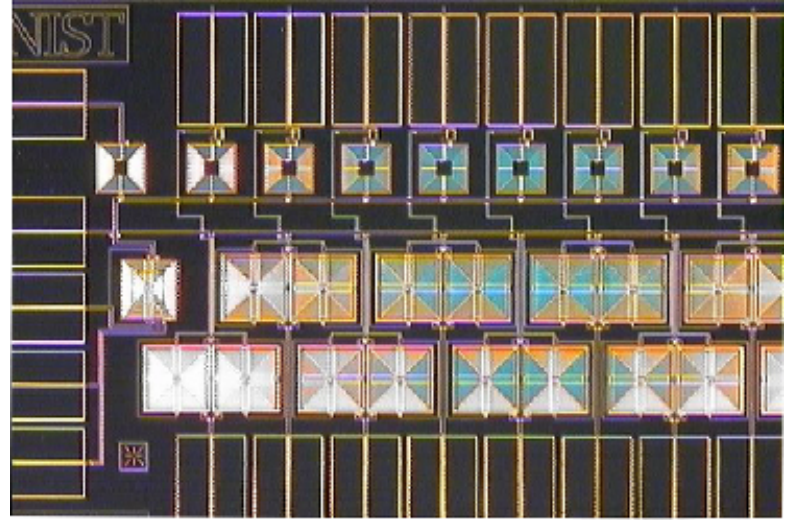

Figure 3. Photograph of part of a 32channel SQUID multiplexer to instrument microcalorimeter arrays 